\title{
EDITORIAL
}

\section{New guidelines for the management of adult lower respiratory tract infections}

\author{
M. Woodhead
}

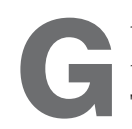
uidelines for the management of adult lower respiratory tract infections (LRTIs) were first published by a Task Force of the European Respiratory Society (ERS) in 1998 [1]. In 2005, a completely revised version was produced, this time by a joint Task Force of the ERS and the European Society for Clinical Microbiology and Infectious Diseases (ESCMID) [2]. They used up-to-date methodology for guideline development, including a transparent and objective systematic literature search strategy, and evidence and recommendation grading [2]. Now, 6 yrs later, a joint Task Force of the two Societies, using the same methodology as in 2005 , has produced a further update of these guidelines incorporating publications through to May 2010. The Task Force included an epidemiologist with expertise in guideline methodology and experts in the specialty areas relevant to LRTI management, including general practice, microbiology, infectious diseases, respiratory medicine, intensive care and public health.

A short version of the guidelines containing only the recommendations has now been published in Clinical Microbiology and Infection [3], with more detailed versions available on each Society's website. The guidelines cover the breadth of adult community-acquired respiratory infection, including prevention (both vaccine- and nonvaccine-related), infections in the community and infections in those admitted to hospital, including pneumonia, exacerbations of chronic obstructive pulmonary disease (COPD) and exacerbations of bronchiectasis. The web versions contain, in addition to guideline recommendations, detailed methodology, background sections on microbial causes, microbial resistance and its clinical relevance, pharmacokinetics, topic discussions relevant to each recommendation, and evidence grading of all references.

It is not the purpose of this editorial to go through aspects of the guideline recommendations; indeed, the topic is vast and the guidelines contain 105 graded recommendations. The guidelines consolidate the recommendations laid out in the 2005 document. While many recommendations have not changed, some have, and in some, the evidence to support the recommendation has changed while the actual recommendation has not. In addition, some new topics have been introduced, such as aspiration pneumonia. It is important to stress that the management of LRTIs includes many aspects, including differentiation from other noninfective causes, decisions about the appropriate place of care, severity assessment, microbial investigation and

CORRESPONDENCE: M. Woodhead, Dept of Respiratory Medicine, Manchester Royal Infirmary, Oxford Road, Manchester M13 9WL, UK. E-mail: mark.woodhead@cmft.nhs.uk prevention, but inevitably, a major focus of interest is likely to be the antibiotic recommendations. Providing recommendations on antibiotic choice is always difficult because of the paucity of high-quality evidence (e.g. good-quality randomised controlled trials, meta-analyses or systematic reviews). This is compounded by differing antibiotic availability in different countries, different traditional prescribing practices and different bacterial resistance rates. However, the microbial causes differ little between countries. For nonsevere LRTIs, especially in the community, we have recognised that there is no good evidence of outcome benefit of one antibiotic type over another, so the recommendations give a wide range of choices, but favour those causing the least harm and those with which there is the greatest prescribing experience. This is supported by at least one more recent study of LRTI community antibiotic prescribing practice [4].

So do we need these guidelines? The aim of guidelines is to improve healthcare practice and, ultimately, outcome. The importance of LRTIs is not in doubt. Cough, usually due to LRTI, is one of the commonest reasons for general practitioner consultation and LRTI is second only to diarrhoeal illness as a cause of episodes of illness in Europe [5]. Community-acquired pneumonia occurs at a rate of 0.9-15.1 cases per 1,000 per year and costs around $€ 10.1$ billion annually [6]. COPD affects some $5 \%$ of the adult population and exacerbations occur, on average, at a rate of 1.2 per person per year [7]. We know little about how LRTIs are managed across Europe, but the little that we know suggests a variability in practice that is not supported by the available evidence on causation and treatment outcomes. We know that there is a huge diversity and excess of antibiotic use [8]. We also know that antimicrobial resistance is a problem that is directly associated with antimicrobial use in the community [8]. Even within countries, antibiotic practice for the same LRTI can vary between hospitals [9, 10], and mortality from community-acquired pneumonia appears to vary between countries [6]. It seems reasonable to suggest that our management of LRTIs could be improved and that guidelines might be one approach to this.

So do guidelines for LRTI improve outcome? Few high-quality studies have addressed this question. However there are many lower quality studies that seem to show a consistent link between guideline use, or concordance, and improved outcomes $[11,12]$. Most such studies are retrospective and focus only on antibiotic use, and it is thus impossible to know whether it is guideline-concordant antibiotic use that is the cause of better outcome, whether this represents a consistent bias in the antibiotic prescribing decision [13], or whether this 
is a marker for another aspect of clinical practice or overall clinical practice that affects outcome. The above studies did not, however, use the ERS/ECSMID guidelines: so are they being read? Certainly by the scientific community it appears, so with 407 citations listed for the 2005 ERS/ESCMID guidelines at the time of writing. Does the practising clinician read them? This is more difficult to answer, but may be the wrong question. In a very recent study of adults with LRTI in the community, antibiotic prescribing was concordant with the ERS/ESCMID guidelines in a high proportion (65\%) [14]. The guidelines may only need to influence national or local policy to have a benefit, so we should perhaps not drill too deeply.

The absence of high-quality evidence to support guideline recommendations is a concern. This is common to many guidelines. In a study of the evidence used in 44 Infectious Disease Society of North America guidelines published between 1994 and 2009, only 16\% were graded as level I, prompting a plea for support for high-quality research to fill the evidence gap [15]. This plea is definitely to be supported. In the new ERS/ ESCMID LRTI guidelines, the proportion of level I evidence remains low, but rose from 21 to $24 \%$ compared with the 2005 document, which is perhaps as much as might be expected in a 6-yr interval. Partly for this reason, the recommendations in the new guideline are not as robust as would be preferred, but the evidence is what it is. It is hoped that the new guideline will help to guide clinical practice and that better evidence will be ready for the next iteration in a few years' time.

\section{STATEMENT OF INTEREST}

A statement of interest for M. Woodhead can be found at www.erj. ersjournals.com/site/misc/statements.xhtml

\section{REFERENCES}

1 ERS Task Force Report. Guidelines for management of adult community-acquired lower respiratory tract infections. Eur Respir J 1998; 11: 986-991.
2 Woodhead M, Blasi F, Ewig S, et al. Guidelines for the management of adult lower respiratory tract infections. Eur Respir J 2005; 26: $1138-1180$.

3 Woodhead M, Blasi F, Ewig S, et al. Guidelines for the management of lower respiratory tract infections. Clin Microbiol Infect 2011; 17: Suppl. 6, 1-24.

4 Butler CC, Hood K, Verheij T, et al. Variation in antibiotic prescribing and its impact on recovery in patients with acute cough in primary care: prospective study in 13 countries. BMJ 2009; 338: b2242.

5 WHO. The Global Burden of Disease: 2004 Update. Geneva, World Health Organization, 2008.

6 Welte T, Torres A, Nathwani D. Clinical and economic burden of community-acquired pneumonia among adults in Europe. Thorax 2010; [Epub ahead of print DOI: 10.1136/thx.2009.129502].

7 Hurst JR, Vestbo J, Anzueto A, et al. Susceptibility to exacerbation in chronic obstructive pulmonary disease. N Engl J Med 2010; 363: 1128-1138.

8 Goossens H, Ferech M, Vander SR, et al. Outpatient antibiotic use in Europe and association with resistance: a cross-national database study. Lancet 2005; 365: 579-587.

9 Capelastegui A, Espana PP, Quintana JM, et al. Management of community-acquired pneumonia and secular trends at different hospitals. Respir Med 2005; 99: 268-278.

10 Kohlhammer Y, Raspe H, Marre R, et al. Antibiotic treatment of community acquired pneumonia varies widely across Germany. J Infect 2007; 54: 446-453.

11 Dambrava PG, Torres A, Vallès $X$, et al. Adherence to guidelines' empirical antibiotic recommendations and community-acquired pneumonia outcome. Eur Respir J 2008; 32: 892-901.

12 Blasi F, Iori I, Bulfoni A, et al. Can CAP guideline adherence improve patient outcome in internal medicine departments? Eur Respir J 2008; 32: 902-910.

13 Paul MF, Nielsen AD, Gafter-Gvili A, et al. The need for macrolides in hospitalised community-acquired pneumonia: propensity analysis. Eur Respir J 2007; 30: 525-531.

14 Wood J, Butler CC, Hood K, et al. Antibiotic prescribing for adults with acute cough/lower respiratory tract infection: congruence with guidelines. Eur Respir J 2011; 38: 112-118.

15 Khan AR, Khan S, Zimmerman V, et al. Quality and strength of evidence of the Infectious Diseases Society of America clinical practice guidelines. Clin Infect Dis 2010; 51: 1147-1156. 